\title{
Mosquitoes minus malaria
}

\author{
If wild populations of the mosquito \\ that transmits malaria were \\ replaced with insects rendered harmless by genetic \\ engineering, the disease could finally be defeated. \\ But that remains a big 'if', as Tom Clarke finds out.
}

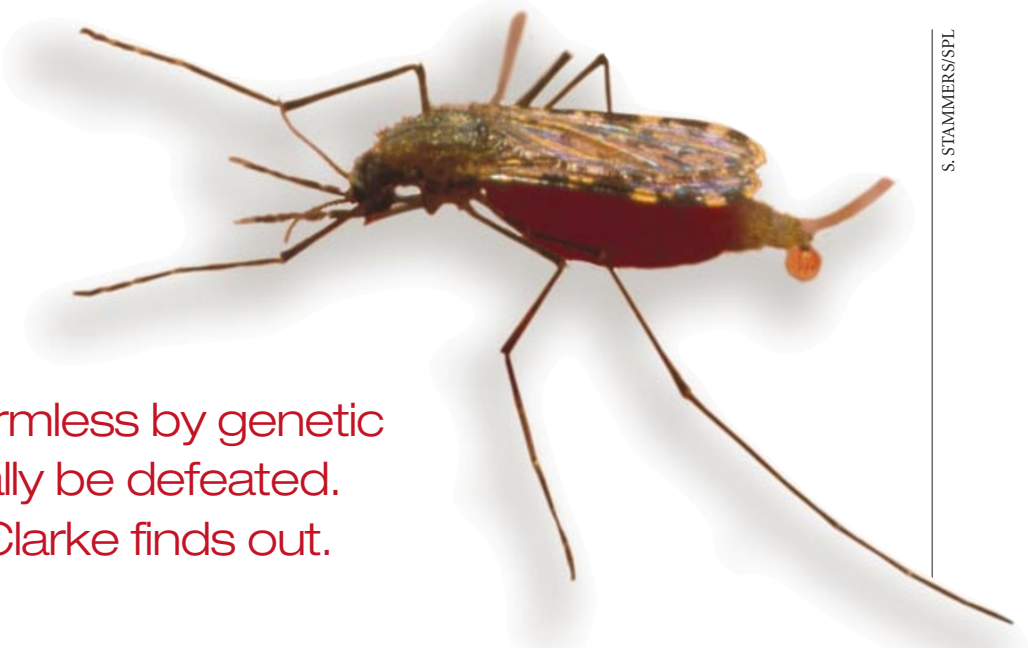

$\mathrm{F}$ or millions of people in sub-Saharan Africa, the whine of Anopheles gambiae is the music of death. This mosquito spreads the malaria parasite Plasmodium falciparum, but if it could be genetically altered to block the cycle of transmission, it would hold no fear. Its bites would be just itchy irritations, its sound merely a mild annoyance.

Following recent advances, genetic engineers are now confident that they can make A. gambiae malaria-proof, or 'refractory'. And the publication in Science this week of the insect's genome sequence ${ }^{1}$ is bound to heighten interest in tinkering with its genetic make-up. But even if a transgenic refractory mosquito can be produced, how can we ensure that it completely infiltrates wild populations? Would the genetic modification remain stable? And what are the public-health implications if the scheme is only a partial success?

Answering these questions will take years of meticulous lab and field research, which at any turn could throw the ambitious scheme off track. Even scientists who support the effort accept that it has to be viewed as a long shot. "I'm not quite ready to hitch my cart to the transgenic wagon," says John Edman, director of the Center for Vector-Borne Diseases at the University of California, Davis.

Some researchers argue that money would be better spent on other approaches to malaria control. But for others, the potential reward remains too great to ignore. Three times in the past two years, most recently in Wageningen, the Netherlands, in June, geneticists, medical entomologists and publichealth experts have gathered to discuss the research that would be required if we are to create a malaria-free future through the release of transgenic refractory A. gambiae.

Just weeks before the Wageningen meeting, a paper published in Nature ${ }^{2}$ illustrated the potential. Researchers led by Marcelo Jacobs-Lorena of Case Western Reserve University in Cleveland, Ohio, showed that adding a synthetic gene for a peptide called SM1 into the related species A. stephensi almost completely disrupted the mosquito's ability to transmit Plasmodium berghei, which causes malaria in mice. The peptide resembles a receptor in the mosquito's gut and salivary glands that is thought to allow the parasite to burrow from one to the other. Flooding the mosquito's body with a similar molecule seems to confuse the parasite, preventing its progress.

Meanwhile, Andrea Crisanti and his colleagues at Imperial College, London, are working on genetic manipulations that direct A. gambiae's immune system against P. falciparum. "Once the best target is identified it would be a matter of months before we have a modified mosquito," Crisanti asserts. Other, more generic approaches are also being considered, such as genetic modifications that render the offspring of the engineered insects sterile.

\section{Jump to it}

But creating genetically modified mosquitoes in the lab is just the start. How can they be made to spread their altered genes through wild A. gambiae populations? It's a tall order, but genetic elements that could fit the bill exist naturally in insect populations. These transposons, or 'jumping genes', copy themselves and move around the genome, being passed down the generations. Once they enter a population, they can spread rapidly through it.

There are many examples, but the most famous is the P-element, discovered in the fruitfly Drosophila melanogaster in the late 1970s. Evidence suggests that the P-element jumped into D. melanogaster from a close relative less than 100 years ago. It is now found in all wild populations of the fly worldwide. So the idea is to slot genes for refractoriness into a suitable transposon, insert this package into a mosquito and then watch it spread.

Here, however, genetic engineers' ambition may be thwarted by natural selection.
Wild A. gambiae have been adapting to their environment for many millions of years. But breed them in the lab for several generations and their evolutionary fitness may plummet. Their ability to compete for food, find mates and avoid predators could all be compromised.

Even if lab-reared strains are continually crossed with vigorous wild-type mosquitoes, the genetic modification itself may also reduce their fitness, as unpublished preliminary work indicates. "There is a cost to mosquitoes of carrying a foreign gene," suggests Peter Billingsley, a molecular physiologist who studies malaria transmission at the University of Aberdeen, UK.

On the positive side, the introduced genes would free the transgenic mosquitoes from the burden of carrying $P$. falciparum. There is some evidence that the parasite has a

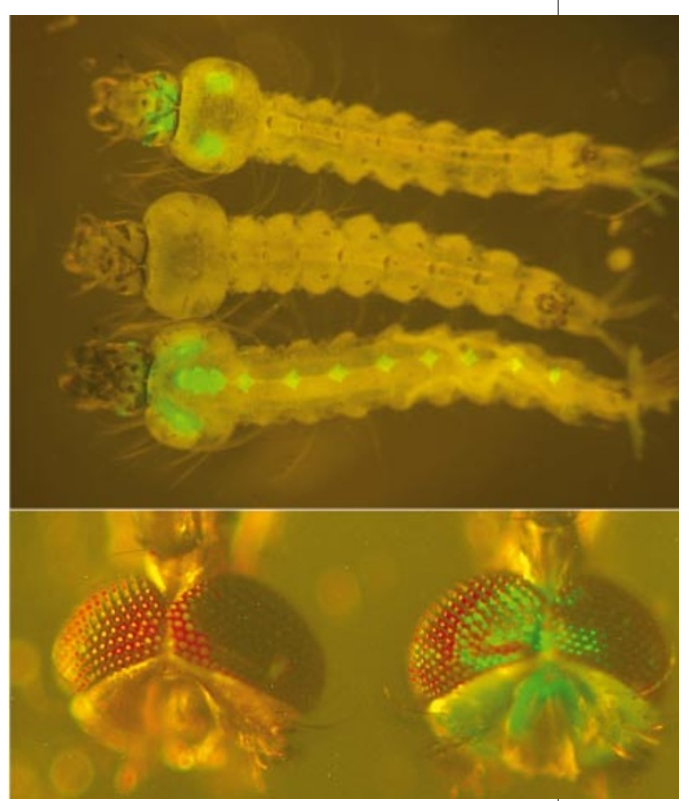

Shining examples: a transgene makes these mosquito larvae (top) and adults glow green. 
detrimental effect on A. gambiae's fitness, but scientists would want to conduct extensive experiments to see whether this would outweigh any fitness deficits before going ahead with field releases.

Population geneticists also warn that the refractoriness genes could become unhitched from their transposon and be left behind. "Once relieved of its burden, the transposon will steam on ahead," says Chris Curtis, a medical entomologist at the London School of Hygiene and Tropical Medicine. And after a particular transposon has spread through a population, it can't be used again.

Perhaps the biggest worry, however, is whether the modification would remain stable in the long term. It could sweep through the global population only to be selected out after a number of years. Alternatively, the malaria parasite might develop resistance to the modification - just as it has to antimalarial drugs. Both scenarios have profound implications for public health. A temporary halt in malaria transmission could result in people losing all natural immunity, rendering the disease even more devastating once the parasite returns. "If malaria were eliminated from Africa for a short period of time, the consequences could be terrible," says Andrew Spielman, a medical entomologist at Harvard University.

\section{Potential pitfalls}

Other concerns are more speculative. As a transposon jumps around the A. gambiae genome, for example, it could disrupt other genes, with unpredictable results. It's extremely unlikely, but what if a transposon gave mosquitoes the ability to transmit other diseases? Changing the relationship between mosquito and malaria parasite also could have unforeseen ecological and evolutionary impacts. Could the parasite adapt quickly enough to use another vector to continue its deadly life cycle? If sterility genes were used to eliminate $A$. gambiae, would other species, which only occasionally bite humans, move in and replace them? Could these new mosquitoes transmit malaria?

Given the catalogue of unknowns, and the potential consequences if the plan were to go awry, experts say that an intensive, multi-stage research programme is needed, similar to the clinical trials of safety and efficacy carried out for experimental drugs. Laboratory studies would first assess the stability, fitness constraints and safety of various genetic modifications. Harmless and well-studied transgenes, such as that for jellyfish green fluorescent protein, could be used at first.

Later on, experiments would move on to the genes that would be used for real, in a controlled environment that mimics the wild as closely as possible. A gigantic green-
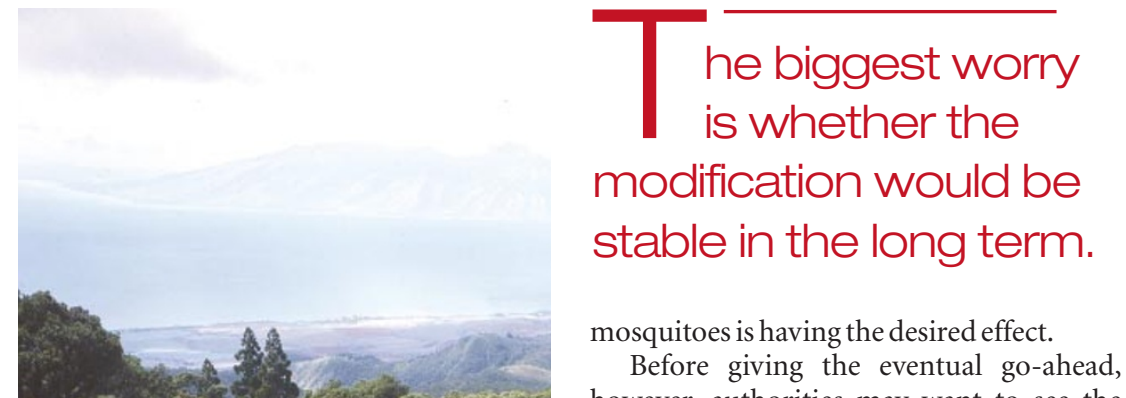

mosquitoes is having the desired effect.

Before giving the eventual go-ahead, however, authorities may want to see the principle demonstrated in the field under circumstances in which the stakes are not so high. Some argue that it would be best to begin on an island with no malaria, working with a mosquito that does not transmit the disease or bite humans. This way, the dynamics of an artificial gene moving through a population could be studied more safely.

\section{Natural setting}

One intriguing idea, championed by Edman, would be to use the geographically isolated Hawaiian islands as a natural laboratory. They are host to the mosquito Culex quinquefasciatus, which transmits a form of malaria to birds. The mosquito was accidentally introduced in the 1820 s and is currently accelerating the decline of the islands' native birds. Releasing transgenic refractory $C$. quinquefasciatus could help to test many of the ecological unknowns while taking

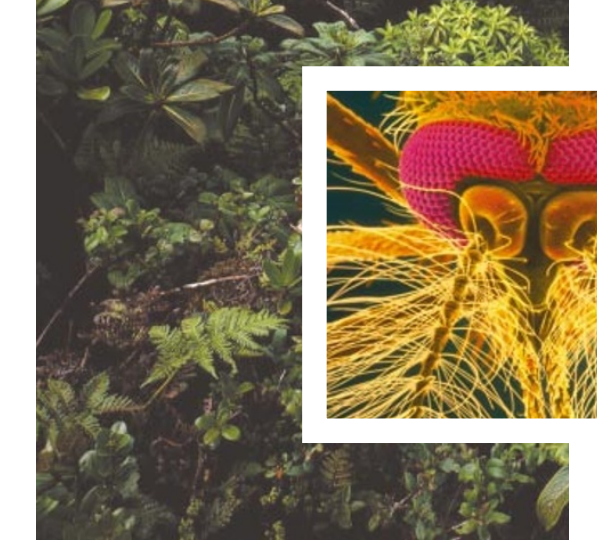

Golden opportunity: Hawaii and its introduced mosquito Culex quinquefasciatus (inset) could provide an ideal test for the transgenic strategy.

house in Kenya, dubbed the 'Malariasphere', could provide a suitable testing ground. Operated by the International Centre of Insect Physiology and Ecology in Nairobi, the Malariasphere contains mock-ups of huts, forest and breeding puddles for mosquitoes. It is currently being used to study the dynamics of A. gambiae populations and their ability to spread malaria.

While such basic research is under way, researchers would need to investigate suitable sites for transgenic releases. Extensive baseline information on local populations of mosquitoes and the burden of malaria in the area would be needed for field workers to be able to tell if a later release of refractory advantage of the excellent advantage of the excellent
scientific infrastructure in Hawaii — which does not exist in many regions afflicted by $P$. falciparum. And if successful, the experiment would help solve a genuine problem.

Conducting the research needed to test the idea of transgenic refractory mosquitoes won't be cheap. But Kate Aultman, an official at the US National Institute of Allergy and Infectious Diseases in Bethesda, Maryland, who is responsible for funding projects on malaria, says that if a reasonable research agenda were constructed, money could be found. "I look forward to getting many proposals," she says.

In any case, say many medical entomologists, the wealth of information on the ecological dynamics of $A$. gambiae populations that would be gained from the research would be valuable in informing other efforts to combat malaria by attacking its vector. "Many of these issues should be high on the agenda," argues Tom Scott, a medical entomologist at the University of California, Davis, "whether we go for an eventual release or not."

\section{Tom Clarke works in Nature's news syndication team.}

\footnotetext{
1. Holt, R. A. et al. Science 298, 129-149 (2002).

2. Ito, J., Ghosh, A., Moreira, L. A., Wimmer, E. A. \& JacobsLorena, M. Nature 417, 452-455 (2002).

3. Anderson, R. A., Knols, B. J. G. \& Koella, J. C. Parasitology 120, 329-334 (1999).

4. Ahmed, A. M. et al. Oikos 97, 371-377 (2002).
} 